\title{
Correction: Wu, S.-L., et al. Simplexins P-S, Eunicellin-Based Diterpenes from the Soft Coral Klyxum simplex. Mar. Drugs 2012, 10, 1203-1211
}

\section{Shwu-Li Wu ${ }^{1,2}$, Jui-Hsin Su ${ }^{3,4}$, Chiung-Yao Huang ${ }^{1}$, Chi-Jen Tai ${ }^{1}$, Ping-Jyun Sung ${ }^{3,4}$, Chih-Chung Liaw ${ }^{1}$ and Jyh-Horng Sheu ${ }^{1,5, *}$}

1 Department of Marine Biotechnology and Resources, National Sun Yat-sen University,

Kaohsiung 804, Taiwan; E-Mails: wusl@webmail.nkmu.edu.tw (S.-L.W.);

betty8575@yahoo.com.tw (C.-Y.H.); jean801023@hotmail.com (C.-J.T.);

ccliaw@mail.nsysu.edu.tw (C.-C.L.)

2 Center of General Studies, National Kaohsiung Marine University, Kaohsiung 811, Taiwan

3 National Museum of Marine Biology \& Aquarium, Pingtung 944, Taiwan;

E-Mails: x2219@nmmba.gov.tw (J.-H.S.); pjsung@nmmba.gov.tw (P.-J.S.)

4 Graduate Institute of Marine Biotechnology, National Dong Hwa University, Pingtung 944, Taiwan

5 Division of Marine Biotechnology, Asia-Pacific Ocean Research Center, National Sun Yat-sen University, Kaohsiung 804, Taiwan

* Author to whom correspondence should be addressed; E-Mail: sheu@mail.nsysu.edu.tw; Tel.: +886-7-5252000 (ext. 5030); Fax: +886-7-5255020.

Received: 17 October 2013 / Accepted: 20 November 2013 / Published: 13 December 2013

We found some errors in our previous published paper [1]. The structure of simplexin Q was found to be the same as klysimplexin C, previously published in Tetrahedron 2009, 65, 7016-7022 [2]. Also, simplexin S and a known compound cladieunicellin G, reported in Chem. Pharm. Bull. 2012, 60, 160-163 [3], have the same structure (see Figure 1). We apologize for any inconvenience caused to the readers by these errors. 
Figure 1. Chemical structures of simplexin Q and simplexin S.

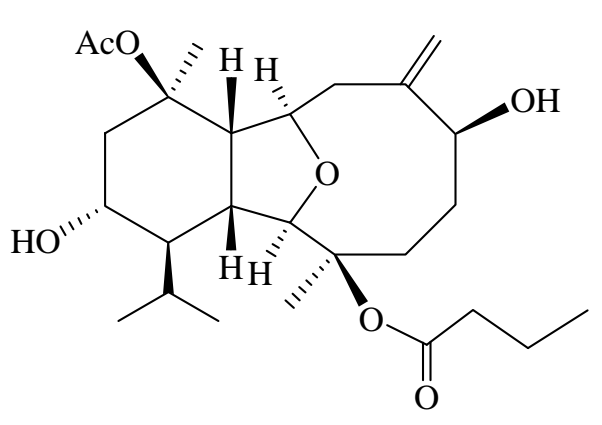

Simplexin Q

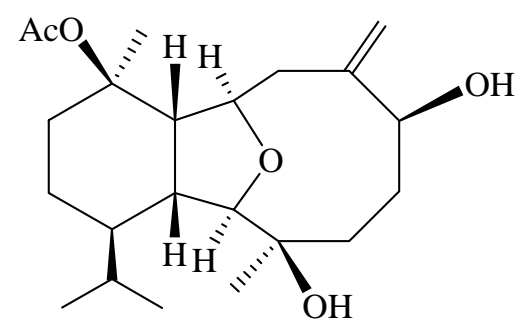

Simplexin S

\section{References}

1. Wu, S.-L.; Su, J.-H.; Huang, C.-Y.; Tai, C.-J.; Sung, P.-J.; Liaw, C.-C.; Sheu, J.-H. Simplexins P-S, eunicellin-based diterpenes from the soft coral Klyxum simplex. Mar. Drugs 2012, 10, 1203-1211.

2. Chen, B.-W.; Wu, Y.-C.; Chiang, M.Y.; Su, J.-H.; Wang, W.-H.; Fan, T.-Y.; Sheu, J.-H. Eunicellin-based diterpenoids from the cultured soft coral Klyxum simplex. Tetrahedron 2009, 65, 7016-7022.

3. Chen, Y.-H.; Hwang, T.-L.; Su, Y.-D.; Chang, Y.-C.; Chen, Y.-H.; Hong, P.-H.; Hu, L.-C.; Yen, W.-H.; Hsu, H.-Y.; Huang, S.-J.; et al. New 6-hydroxyeunicellins from a soft coral Cladiella sp. Chem. Pharm. Bull. 2012, 60, 160-163.

(C) 2013 by the authors; licensee MDPI, Basel, Switzerland. This article is an open access article distributed under the terms and conditions of the Creative Commons Attribution license (http://creativecommons.org/licenses/by/3.0/). 